\title{
Pingmu decoction enhances apoptosis of orbital adipocytes derived from patients with Graves' ophthalmophathy
}

\author{
HONG LI, YAQIONG WANG and RONGJUAN XU \\ Department of Endocrinology, Longhua Hospital, Shanghai, P.R. China
}

Received April 10, 2012; Accepted September 6, 2012

DOI: $10.3892 / \mathrm{mmr} .2012 .1080$

\begin{abstract}
Graves' ophthalmopathy (GO), an autoimmune disease, has been demonstrated to result from an increased volume of orbital contents, including adipose, connective and extraocular muscle tissues. In our previous study, we showed that Pingmu decoction is capable of alleviating GO progression. In this study, to further investigate the underlying mechanism(s), we examined the effects of Pingmu decoction-containing serum on the proliferation and apoptosis of preadipocytes and adipocytes derived from the orbital adipose tissue of GO patients. Our data demonstrate for the first time that Pingmu decoction-containing serum significantly reduces preadipocyte proliferation and increases adipocyte apoptosis as measured by MTT assay and Annexin V/FITC staining, respectively. Moreover, Pingmu decoction elevated the levels of cleaved caspase-3, 8 and 9 and decreased the levels of cell cycle-related genes compared to those of the control. In addition, cell cycle arrest at the G0/G1 phase was observed following Pingmu decoction treatment. This study shows a mechanism by which Pingmu decoction serves as an effective GO medicine by downregulating preadipocyte proliferation and increasing adipocyte apoptosis.
\end{abstract}

\section{Introduction}

Graves' disease (GD), the most common cause of hyperthyroidism, usually presenting itself during early adolescence, is an autoimmune disease in which the thyroid is overactive, producing an excessive amount of thyroid hormones. It has been demonstrated that hyperactivation of the thyroid is caused by thyroid autoantibodies [thyroid stimulating hormone (TSH; also known as thyrotropin) receptor (TSHR)-Ab)] that activate the TSHR, thereby stimulating thyroid hormone synthesis and secretion, as well as thyroid growth (1). Hyperthyroidism may cause a marked combination of neuropsychological and physical signs and symptoms. In addition to hyperthyroidism,

Correspondence to: Dr Hong Li, Department of Endocrinology, Longhua Hospital, No. 725 South Wanping Road, Shanghai 200030, P.R. China

E-mail: dlbtzh@126.com

Key words: Pingmu decoction, apoptosis, adipocytes, Graves' ophthalmophathy clinical involvement of the eyes, Graves' ophthalmopathy (GO), develops in 25 to $50 \%$ of individuals with GD (2). Although certain patients with GO experience only mild ocular discomfort, approximately 5\% have severe ophthalmopathy, including excessive chemosis, proptosis or even loss of vision (3). The clinical symptoms and signs of GO can be explained mechanically by the discrepancy between the increased volume of the swollen orbital tissues and the fixed volume of the bony orbit (2). The expanded orbital tissues displace the globe forward and impede venous outflow from the orbit. These changes, combined with the local production of cytokines and other mediators of inflammation, result in pain, proptosis, periorbital edema, conjunctival injection and chemosis.

For GO treatment, the first step usually involves the regulation of thyroid hormone levels. Topical lubrication of the ocular surface is used to avoid corneal damage caused by exposure. Tarsorrhaphy is an alternative option when the complications of ocular exposure cannot be avoided solely with the eye drops. Corticosteroids are efficient in reducing orbital inflammation; however, the benefits cease after discontinuation. Corticosteroid treatment is also limited due to the many sideeffects (4). Radiotherapy is an alternative option to reduce acute orbital inflammation (5). However, there remains controversy surrounding its efficacy. A simple way of reducing inflammation is smoking cessation, as pro-inflammatory substances are found in cigarettes (6). Surgery may also be performed to decompress the orbit, to improve the proptosis and to address the strabismus causing diplopia. Surgery is performed once the patient's disease has been stable for at least 6 months. Eyelid surgery is the most common surgery performed on $\mathrm{GO}$ patients.

Several novel approaches for the treatment of GO follow logically from the current understanding of pathogenesis. Studies from several laboratories underline the pathogenic role of both Th-1-type and macrophage-derived cytokines in early disease pathogenesis (7). Therefore, monoclonal antibodies that target pro-inflammatory cytokines and chemokines may hold particular promise. Specifically, biological agents that block the tumor necrosis factor (TNF) (infliximab, adalimumab or etanercept) or interleukin (IL)-1 receptor (anakinra) are attractive theoretical choices. These agents are effective in rheumatoid arthritis and Crohn's disease therapy, and they are under investigation for the treatment of diverse conditions, such as uveitis, sarcoidosis, interstitial lung disease, graft-versus-host disease and Sjögren's syndrome. However, although these drugs have revolutionized the treatment of several immune-mediated 
inflammatory diseases, there is growing evidence that TNF inhibition is associated with serious side-effects. Of particular concern are several case reports of serious infections, including the reactivation of Mycobacterium tuberculosis (8). In addition, lymphoma, demyelinating disorders, hepatotoxicity, aplastic anemia and lupus-like syndrome have been described in association with TNF antagonists. In the future, the application of knowledge concerning genetic variability and TNF/TNF receptor polymorphisms may aid in the targeting of anti-TNF therapy for patients most likely to benefit and least likely to have adverse effects.

A study by Peyster et al showed that there was excellent correlation between proptosis and percentage fat volume, supporting the contention that increased orbital fat is responsible for proptosis (9). In another study by Kumar et al, researchers determined whether the expanded adipose tissue volume may be in part attributable to de novo adipogenesis by measuring the levels of mRNA encoding leptin, adiponectin, peroxisome proliferator-activated receptor (PPAR), preadipocyte factor- 1 and TSHR sgenes in orbital adipose tissues from GO patients and normal individuals, and in orbital preadipocyte cultures derived from GO patients and normal subjects using quantitative real-time RT PCR. The results showed increased leptin, adiponectin, PPAR and TSHR expression in GO compared with normal orbital tissue samples, with positive correlations in the GO tissues between TSHR and leptin, adiponectin and PPAR (10). Th in vitro differentiation of GO and normal preadipocytes resulted in enhanced adiponectin, leptin and TSHR expression, with greater expression of the latter 2 genes in the GO cultures. These results suggest that de novo adipogenesis within orbital tissues with parallel enhanced expression of TSHR may be important in the pathogenesis of $\mathrm{GO}$, and that potential therapies for GO may include the inhibition of the adipogenic pathway. Preadipocytes may also be separated from the orbital adipose tissue of GO patients and become differentiated into mature adipocytes in vitro, indicating that preadipocyte differentiation may occur (11).

Pingmu decoction is composed of a variety of Chinese herbal medicines (Astragalus $30 \mathrm{~g}$, Herba epimedii $15 \mathrm{~g}$, root of red rooted Salvia $15 \mathrm{~g}$, Semen brassicae $15 \mathrm{~g}$, Plantago seed $15 \mathrm{~g}$, Oldenlandia diffusa $30 \mathrm{~g}$ ). In our previous study (12), we demonstrated that Pingmu decoction effectively alleviated GO progression. In this study, we investigated the underlying molecular mechanisms by determining the effect of Pingmu decoction on adipocyte proliferation and apoptosis.

\section{Materials and methods}

Cell culture. GO orbital adipose tissue samples were minced and placed directly in plastic culture dishes, allowing preadipocyte fibroblasts to proliferate as described previously (13). Cells were propagated in medium 199 containing $20 \%$ fetal bovine serum (FBS; HyClone Laboratories, Inc., Logan, UT, USA), penicillin $(100 \mathrm{U} / \mathrm{ml})$ and gentamicin $(20 \mu \mathrm{g} / \mathrm{ml})$ in a humidified $5 \% \mathrm{CO}_{2}$ incubator at $37^{\circ} \mathrm{C}$ and were maintained in $80-\mathrm{mm}^{2}$ flasks with medium 199 containing $10 \%$ FBS and antibiotics. To initiate adipocyte differentiation, orbital cells were grown to confluence in 6-well plates in medium 199 with 10\% FBS. Differentiation was carried out as reported previously (11); cultures were changed to serum- free DMEM/Ham's F-12 (1:1; Sigma-Aldrich Corp., St. Louis, MO, USA) supplemented with biotin $(33 \mu \mathrm{m})$, pantothenic acid $(17 \mu \mathrm{m})$, apotransferrin $(10 \mu \mathrm{g} / \mathrm{ml})$, T3 $(0.2 \mathrm{~nm})$, insulin (1 $\mu \mathrm{m})$, carbaprostacyclin $(0.2 \mu \mathrm{m}$; Calbiochem, La Jolla, CA, USA) and, for the first 4 days only, dexamethasone $(1 \mu \mathrm{m})$ and isobutylmethylxanthine $(0.1 \mathrm{~mm})$. The differentiation protocol was continued for 10 days, during which time the medium was replaced every 3-4 days. Undifferentiated cultures were derived from fibroblasts obtained from the orbital tissues of the same patients and were maintained for the same period of time in medium lacking several of the components necessary for complete adipocyte differentiation (i.e., carbaprostacyclin, dexamethasone and isobutylmethylxanthine).

Rat treatment and serum preparation. Male Sprague-Dawley (SD) rats (8 rats for each group) were fed Pingmu decoction or other combinations of ingredients twice a day for 5 consecutive days: Pingmu decoction; Astragalus; Herba epimedii; root of red-rooted Salvia + Semen brassicae + Plantago seed + Oldenlandia diffusa; Astragalus + root of red-rooted Salvia + Semen brassicae + Plantago seed + Oldenlandia diffusa; Astragalus + Herba epimedii, Herba epimedii + root of red-rooted Salvia + Semen brassicae + Plantago seed + Oldenlandia diffusa. Serum was drawn from the abdominal artery $1 \mathrm{~h}$ following the final administration and heat inactivated

Cell apoptosis detection. Adipocytes from the different treatment groups were trypsinized, collected, washed and then stained with Annexin V-FITC and propidium iodide (PI) (BD Biosciences, Heidelberg, Germany) for $10 \mathrm{~min}$ at $4^{\circ} \mathrm{C}$ in the dark according to the manufacturer's instructions. Apoptotic cells were determined by flow cytometry analysis (FACScan; BD Biosciences). The extent of apoptosis was quantified as the percentage of Annexin V-positive cells.

Cell cycle analysis. Following the indicated treatments, adipocytes were harvested and washed twice with $1 \mathrm{X}$ PBS and fixed in $70 \%$ ethanol at $-20^{\circ} \mathrm{C}$ for $16 \mathrm{~h}$. The fixed cells were collected, washed twice with PBS and suspended in PBS containing $10 \mu \mathrm{g} / \mathrm{ml}$ PI (Sigma-Aldrich) and $100 \mu \mathrm{g} / \mathrm{ml}$ RNase A, then incubated at $4{ }^{\circ} \mathrm{C}$ for at least $30 \mathrm{~min}$ avoiding light in order to eliminate the intracellular RNA. Cell cycle distribution was determined using FACSCalibur flow cytometer (FACScan; BD Biosciences).

Western blot analysis. Cells were lysed directly on the culture dishes using lysis buffer $(50 \mathrm{mM}$ Tris- $\mathrm{HCl}, 150 \mathrm{mM} \mathrm{NaCl}$, $0.02 \% \mathrm{NaN}_{3}, 1 \%$ Triton X-100, $1 \mathrm{mM}$ PMSF, $1 \mu \mathrm{g} / \mathrm{ml}$ aprotinin and $1 \mu \mathrm{g} / \mathrm{ml}$ leupeptin). The protein concentration was determined by the Bradford assay kit (Bio-Rad Laboratories, Hercules, CA, USA). Equal amounts of total protein were subjected to $10 \%$ SDS-PAGE and then transferred onto PVDF membranes. Following overnight blocking with 5\% non-fat milk at $4^{\circ} \mathrm{C}$, the membranes were incubated with primary antibodies as follows: anti-caspase-3, anti-caspase-8, anti-caspase-9 anti-cyclin D1, anti-cyclin E1, anti-CDK4, anti-Bcl-2 (Santa Cruz Biotechnology, Santa Cruz, CA, USA) and anti-GAPDH antibodies (BD Biosciences) overnight at $4^{\circ} \mathrm{C}$. The membranes were then incubated with secondary antibody conjugated to horseradish peroxidase (Jackson 


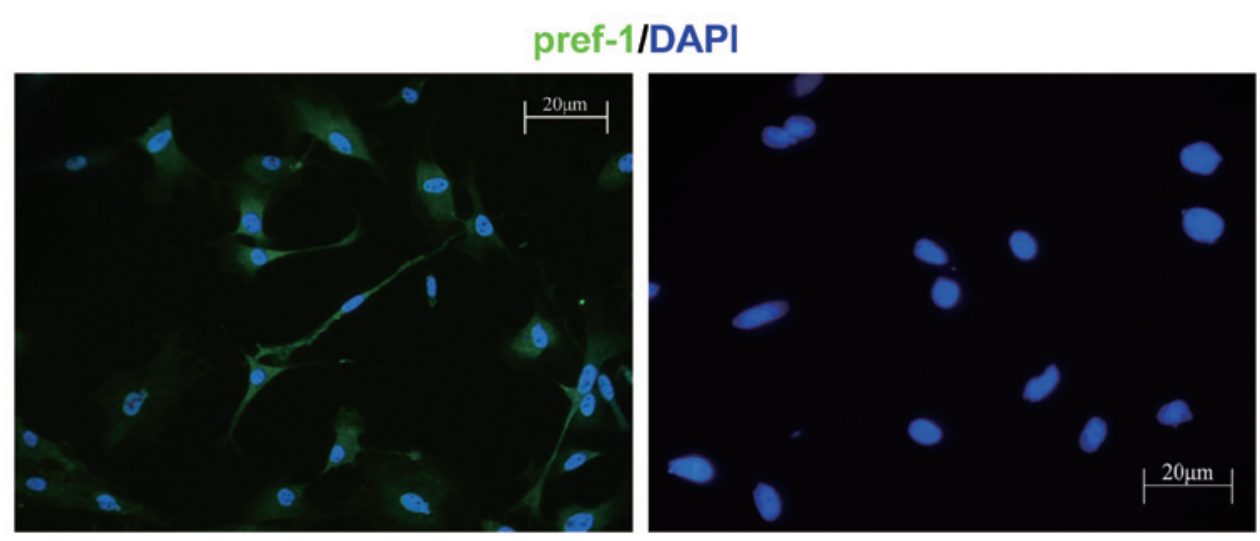

Figure 1. Preadipocyte immunofluorescence images with Pref-1 antibody. Preadipocytes derived from Graves' ophthalmopathy patients were stained with Pref-1 antibody and FITC-conjugated secondary antibody and observed under a fluorescence microscope. Representative images are shown.

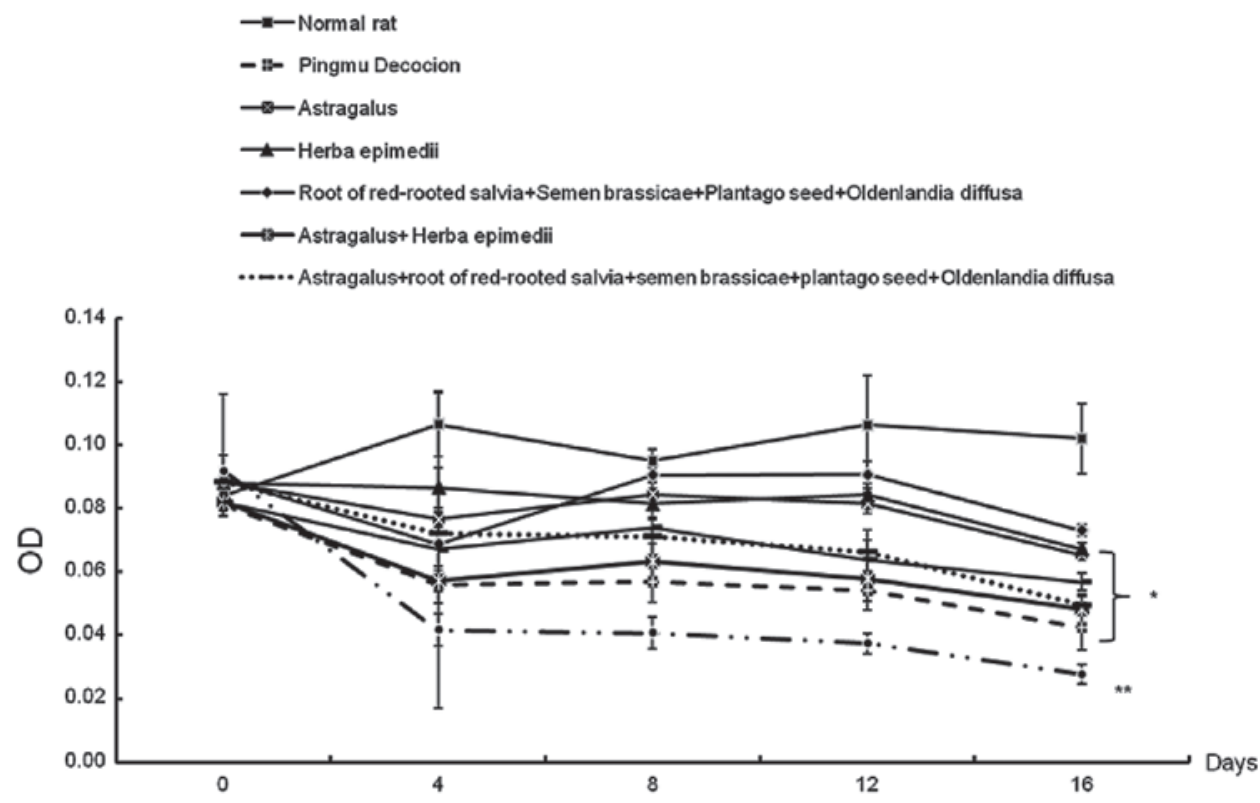

Figure 2. Pingmu decoction reduced preadipocyte proliferation. Preadipocytes were treated with serum obtained from the different groups of rats and cultured for 4, 8, 12 and 16 days. Cell viability was measured by MTT assay. Each dot represents the results from 3 experiments. ${ }^{*} \mathrm{P}<0.05,{ }^{* *} \mathrm{P}<0.05$

ImmunoResearch, West Grove, PA, USA). The protein was visualized using ECL western blotting detection reagents and was then analyzed through scanning densitometry using the Tanon Image system.

Indirect immunofluorescence. Isolated preadipocytes were cultured for $24 \mathrm{~h}$ and fixed with $4 \%$ formaldehyde for $15 \mathrm{~min}$. Cells were washed 3 times with PBS and blocked with 5\% BSA in PBS prior to Pref-1 primary antibody (Santa CruzBiotechnology) incubation. Cells were then stained with FITC-conjugated secondary antibody (JacksonImmunoResearch) and observed under a fluorescence microscope.

Statistical analysis. All experiments were performed in duplicate or triplicate. The data were treated using one-way ANOVA to determine statistically significant differences. $\mathrm{P}<0.05$ was considered to indicate significance and is shown by asterisks in the figures.

\section{Results}

Preadipocyte separation and identification. Orbital adipose tissues were removed from $\mathrm{GO}$ patients and preadipocytes were isolated using a previously published method (13). Following 5 to 10 days in culture, the preadipocytes were elongated cells with the appearance of fibroblasts. Immunofluorescence assays with Pref- 1 antibody were carried out to identify these cells and to determine the purity. After examination under a fluorescence microscope, we were able to show that almost all cells stained positive, indicating that these cells were preadipocytes (Fig. 1).

Effects of Pingmu decoction on preadipocyte proliferation. In order to determine the effect of Pingmu decoction on preadipocyte proliferation, we incubated preadipocytes with serum from rats fed with Pingmu decoction or other combinations of ingredients and examined the cell cycle progression by MTT 

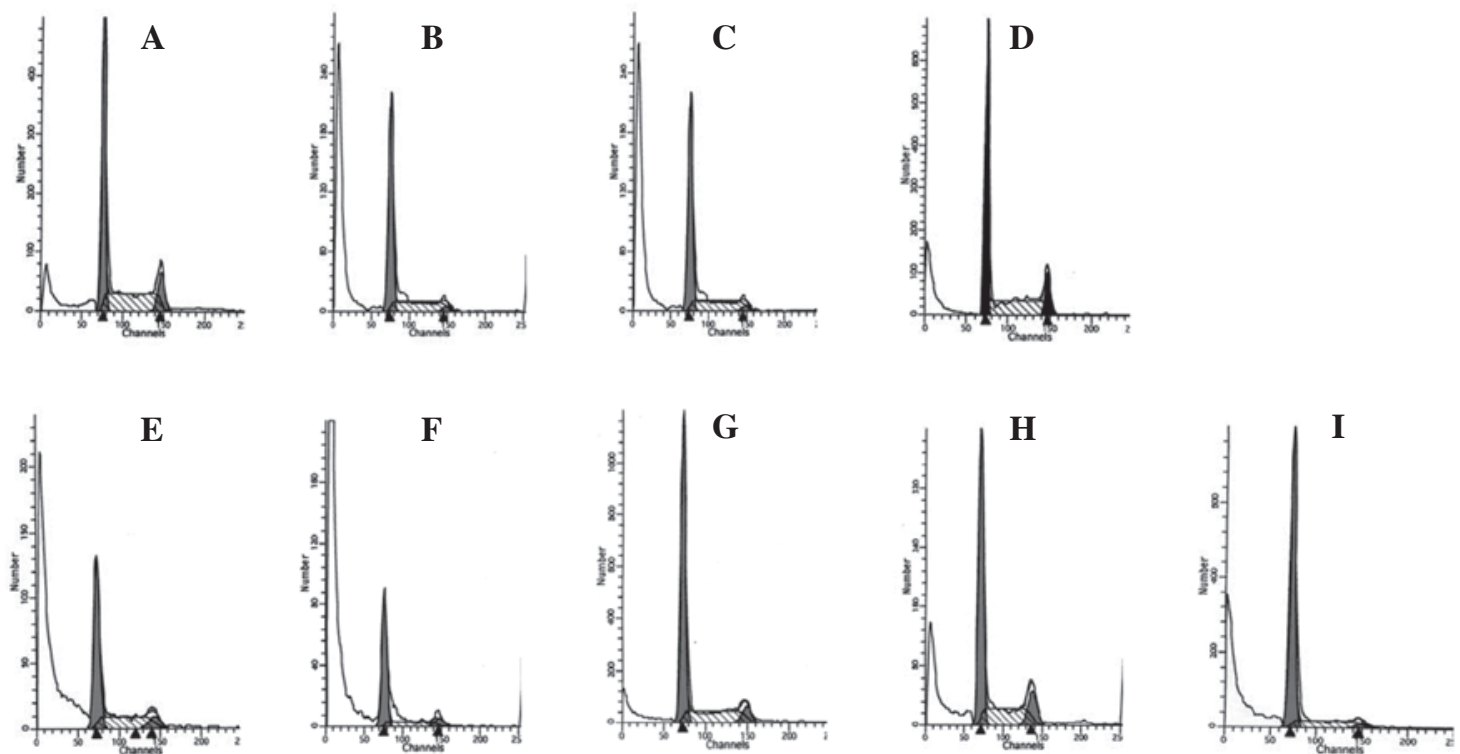

Figure 3. Pingmu decoction serum caused cell cycle arrest at the G0/G1 phase. Adipocytes were treated with serum from the different groups of rats and cultured for 2 days, then stained with propidium iodide and RNase prior to analysis by flow cytometry. Experiments were performed 3 times and representative images are shown. (A) Normal rat; (B) Pingmu decoction; (C) Astragalus; (D) Herba epimedii; (E) Root of red-rooted Salvia + Semen brassicae + Plantago seed + Oldenlandia diffusa; (F) Astragalus + root of red-rooted Salvia + Semen brassicae + Plantago seed + Oldenlandia diffusa; (G) Astragalus + Herba epimedii; (H) Herba epimedii + root of red-rooted Salvia + Semen brassicae + Plantago seed + Oldenlandia diffusa; (I) $30 \mu$ gml dexamethasone acetate injection.

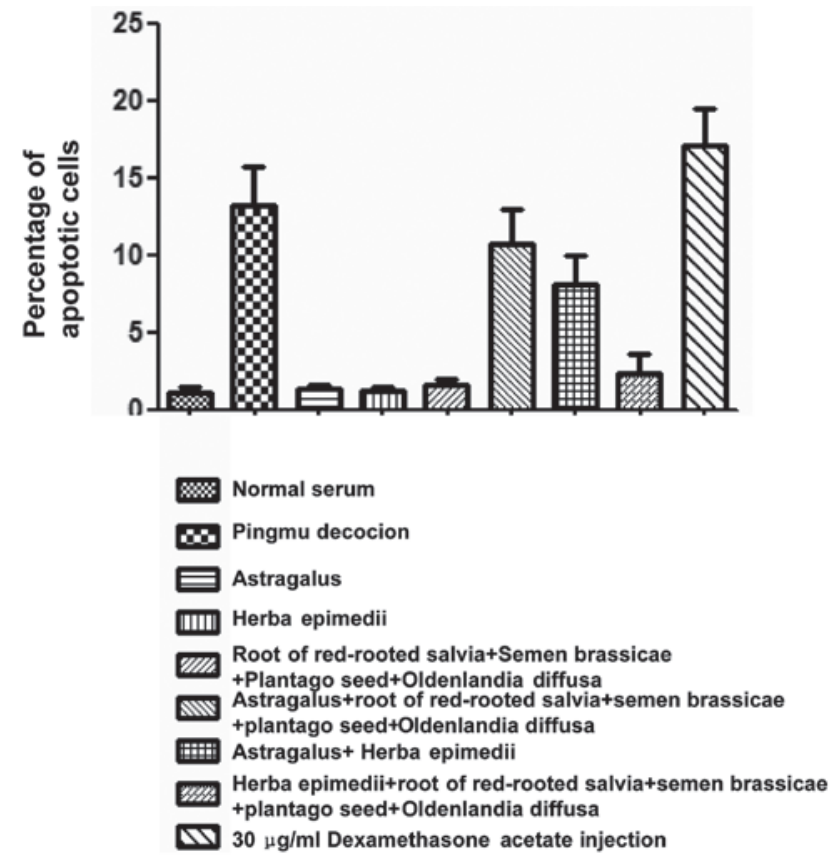

Figure 4. Pingmu decoction enhanced adipocyte apoptosis. Adipocytes were treated with serum from the different groups of rats and cultured for 2 days, then analyzed by flow cytometry using Annexin V and PI staining. Annexin V-positive PI-negative apoptotic cells were counted. Experiments were performed 3 times. PI, propidium iodide.

assay at different time-points. As demonstrated in Fig. 2, Pingmu-containing serum, along with serum from the other groups of rats fed with the other combinations of ingredients, significantly reduced preadipocyte proliferation.

Effects of Pingmu decoction on the cell cycle and apoptosis of adipocyte. To assess the effects of Pingmu decoction on

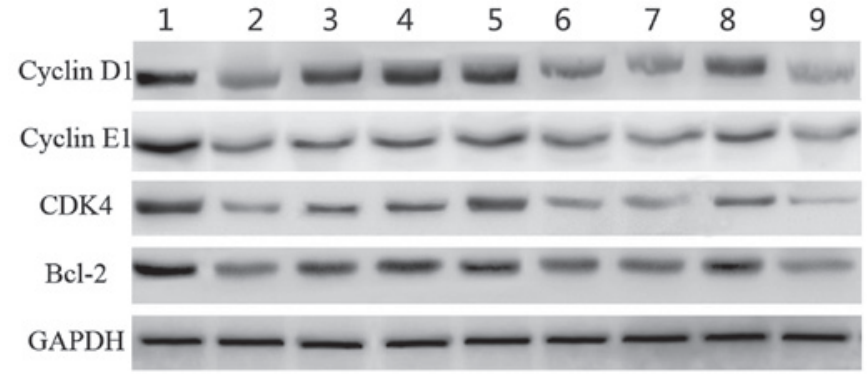

Figure 5. Pingmu decoction inhibited the expression of cell cycle related genes. Adipocytes were treated with serum from the different groups of rats and cultured for 2 days, and then the total proteins of the cells were extracted. Western blot analysis was performed to determine the expression of cell cycle-related genes, including cyclin D1, cyclin E1, CDK4 and Bcl-2. GAPDH served as the internal control. Lane 1, normal rat; lane 2, Pingmu decoction; lane 3, Astragalus; lane 4, Herba epimedii; lane 5, Root of red-rooted Salvia + Semen brassicae + Plantago seed + Oldenlandia diffusa; lane 6, Astragalus + root of red-rooted Salvia + Semen brassicae + Plantago seed + Oldenlandia diffusa; lane 7, Astragalus + Herba epimedii; lane 8, Herba epimedii + root of red-rooted Salvia + Semen brassicae + Plantago seed + Oldenlandia diffusa; lane 9,30 $\mu \mathrm{gml}$ dexamethasone acetate injection.

the cell cycle and apoptosis of adipocytes, we first detected cell cycle distribution and Annexin V/FITC staining by flow cytometry. These experiments showed that Pingmu decoctioncontaining serum caused an increase in the G1/G0 cell percentage at a level similar to dexamethasone (Fig. 3) and enhanced Annexin V staining levels (Fig. 4). These results were confirmed by western blot analysis, which showed that Pingmu decoction serum had a similar effect as dexamethasone on reducing the expression of the cell cycle-related genes (Fig. 5). The results indicated that Pingmu had a significant effect on the inhibition of preadipocyte proliferation. We then examined the cleavage of caspase- 3,8 and 9 by immunobloting, 

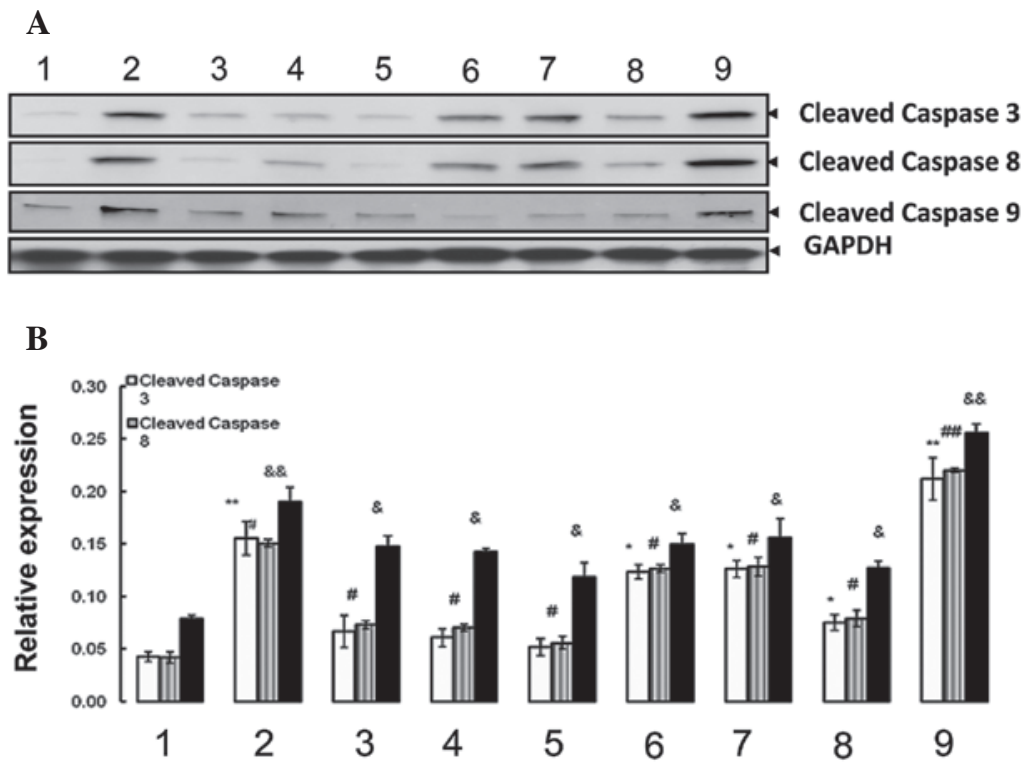

Figure 6. Pingmu decoction elevated the levels of cleaved caspase-3,8 and 9. Adipocytes were treated with serum from the different groups of rats and cultured for 2 days, then cell lysed for the detection of cleaved caspase-3, 8 and 9 by (A) western blot analysis. (B) The optical density values of corresponding protein bands were semi-quantified by IPP 6.0 software. Experiments were performed 3 times and representative images are shown. Lane 1, normal rat; lane 2, Pingmu decoction; lane 3, Astragalus; lane 4, Herba epimedii; lane 5, root of red-rooted Salvia + Semen brassicae + Plantago seed + Oldenlandia diffusa; lane 6 , Astragalus + root of red-rooted Salvia + Semen brassicae + Plantago seed + Oldenlandia diffusa; lane 7, Astragalus + Herba epimedii; lane 8, Herba epimedii + root of red-rooted Salvia + Semen brassicae + Plantago seed + Oldenlandia diffusa; 9, $30 \mu$ gml dexamethasone acetate injection. GAPDH was used as the inner control to show the equal amount of loaded protein. ${ }^{*} \mathrm{P}<0.05,{ }^{* *} \mathrm{P}<0.01$, Compared to normal group in expression of cleaved caspase 3 ; ${ }^{\&} \mathrm{P}<0.05,{ }^{\&} \& \mathrm{P}<0.01$, Compared to normal group in expression of cleaved caspase $8 ;{ }^{\#} \mathrm{P}<0.05,{ }^{\# \#} \mathrm{P}<0.01$, Compared to normal group in expression of cleaved caspase 9.

discovering that Pingmu decoction-containing serum, with a similar efficacy as dexamethasone, prompted the cleavage of caspase-3, 8 and 9 (Fig. 6). These data suggest that Pingmu decoction significantly increases adipocyte apoptosis.

\section{Discussion}

GO is an autoimmune condition most frequently associated with GD (12). In GD, TSHR is the target of stimulating autoantibodies (TSAB), which mimic the action of TSH by increasing intracellular cyclic adenosine monophosphate (cAMP) levels (2). This leads to increased thyrocyte function and growth, independent of the hypothalamic-pituitary-thyroid axis. The pathogenesis of GO is less clear. The majority of the signs and symptoms may be explained by the increase in volume of the orbital contents. The extraocular muscles become grossly enlarged, mostly due to edema. There are few microscopically visible changes in the myocytes, although in advanced disease they become heavily fibrosed. Apart from the edema, 2 other mechanisms increase orbital volume: the production of glycosaminoglycans (by orbital fibroblasts), which absorb water and swell, and hyperplasia of the adipose tissue. In combination, these cause proptosis and compression of the optic nerve, which may result in diplopia and in extreme cases, loss of sight.

Recent studies show that adipogenesis is a major contributor to GO progression (3). Adipose tissue volume increases through a combination of increased cell number (hyperplasia) and expanded cell size (hypertrophy). New adipocyte formation plays an ongoing role in adipose tissue enlargement throughout life. A previous study demonstrated that cultures derived from human orbital adipose/connective tissue contain adipocyte precursor cells (comprising 5-10\% of the total), capable of differentiating into lipid-filled adipocytes when cultured under conditions known to stimulate adipogenesis in fibroblasts from other sites. Adipogenesis is a complex process associated with the activation of several adipocyte-specific genes, including leptin, adiponectin and PPAR, and the inhibition of the preadipocyte gene pref-1. PPAR is a nuclear hormone receptor that is highly expressed in adipose tissue. The activation of this receptor is critical for the complex processes of adipogenesis, and several ligands of this receptor that have profound effects on this process, as well as on insulin sensitivity, have been developed. Leptin is a protein produced and secreted exclusively by mature adipocytes.

Similarly, adiponectin is a recently identified, adipose tissuederived, soluble protein produced solely by mature adipocytes. This protein has important metabolic effects related to whole body insulin sensitivity and also possesses anti-atherogenic properties. Serum levels of adiponectin decrease with obesity and are higher in females than in males. Both adiponectin expression and secretion are stimulated by activators of PPAR. It has been demonstrated that there is a significantly increased expression of all 3 gene markers of adipocyte differentiation (leptin, adiponectin and PPAR) in orbital adipose tissue from patients with GO compared with normal orbital tissue. In addition, the expression of each of these genes correlated positively with TSHR gene expression. A study by Kumar et al found higher levels of leptin and adiponectin, genes produced exclusively by mature adipocytes, in uncultured orbital adipose tissue specimens from patients with GO compared with normal orbital tissue specimens (10). These results suggest that there may be a relatively greater number of mature adipocytes in GO than in normal orbital tissues. This may result from the stimulation of adipogenesis in orbital preadipocytes by some 
unknown factor present in GD. Naturally, they suggest that the inhibition of orbital adipogenesis by the antagonism of various components of the adipogenic pathway may be of benefit in the treatment of GO.

As for the treatment of GO, corticosteroids are efficient in reducing orbital inflammation; however, the benefits cease after discontinuation. Corticosteroid treatment is also limited due to the many side-effects. Radiotherapy is an alternative option to reduce acute orbital inflammation. However, there remains controversy surrounding its efficacy. The lack of efficient medication with minimal side-effects led us to search for an alternative therapy. We turned to traditional Chinese medicine.

In the present study, we examined the effect of Pingmu decoction or other combinations of ingredients on the proliferation and apoptosis of preadipocytes and adipocytes derived from fat tissue of GO patients. We successfully separated preadipocytes and induced adipocyte differentiation in conditional medium. We then treated those cells with serum from rats feeding on Pingmu decoction or other combinations of ingredients. We observed a decrease in the proliferation of preadipocytes. We also showed that Pingmu decoction-containing serum has an effect on reducing the expression of cell cycle-related genes. These results suggest that Pingmu decoction inhibits preadipocyte proliferation. By immunoblotting, we observed the increase in the apoptosis of adipocytes treated with Pingmu-containing serum, determined by enhanced Annexin V staining, cell cycle arrest at the G0/G1 phase and increased levels of cleaved caspase-3, 8 and 9. Moreover, Astragalus + root of red-rooted Salvia + Semen brassicae + Plantago seed + Oldenlandia diffusa serum, and Astragalus + Herba epimedii serum showed similar effects on the inhibiton of proliferation of preadipocytes and the enhancement of adipocyte apoptosis. Collectively, these data support the conclusion that Pingmu decoction is capable of inhibiting preadipocyte proliferation and enhancing adipocyte apoptosis and may potentially be applied to clinical practice.

\section{Acknowledgements}

This project is supported by Grants from the National Natural Science Foundation of China (No. 81072793) and Key Project of Scientific Research and Innovation of Shanghai Education Commission (No. 11ZZ114).

\section{References}

1. Bahn RS: Graves' ophthalmopathy. N Engl J Med 362: 726-738, 2010.

2. Bahn RS and Heufelder AE: Pathogenesis of Graves' ophthalmopathy. N Engl J Med 329: 1468-1475, 1993.

3. Garrity JA and Bahn RS: Pathogenesis of graves ophthalmopathy: implications for prediction, prevention, and treatment. Am J Ophthalmol 142: 147-153, 2006.

4. Bartalena L, Marcocci C, Bogazzi F, Manetti L, Tanda ML, Dell'Unto E, Bruno-Bossio G, Nardi M, Bartolomei MP, Lepri A, Rossi G, Martino E and Pinchera A: Relation between therapy for hyperthyroidism and the course of Graves' ophthalmopathy. N Engl J Med 338: 73-78, 1998.

5. Behbehani R, Sergott RC and Savino PJ: Orbital radiotherapy for thyroid-related orbitopathy. Curr Opin Ophthalmol 15: 479-482, 2004.

6. Stan MN and Bahn RS: Risk factors for development or deterioration of Graves' ophthalmopathy. Thyroid 20: 777-783, 2010.

7. Kumar S and Bahn RS: Relative overexpression of macrophagederived cytokines in orbital adipose tissue from patients with graves' ophthalmopathy. J Clin Endocrinol Metab 88: 4246-4250, 2003.

8. Ellerin T, Rubin RH and Weinblatt ME: Infections and anti-tumor necrosis factor alpha therapy. Arthritis Rheum 48: 3013-3022, 2003.

9. Peyster RG, Ginsberg F, Silber JH and Adler LP: Exophthalmos caused by excessive fat: CT volumetric analysis and differential diagnosis. AJR Am J Roentgenol 146: 459-464, 1986.

10. Kumar S, Coenen MJ, Scherer PE and Bahn RS: Evidence for enhanced adipogenesis in the orbits of patients with Graves' ophthalmopathy. J Clin Endocrinol Metab 89: 930-935, 2004

11. Crisp M, Starkey KJ, Lane C, Ham J and Ludgate M: Adipogenesis in thyroid eye disease. Invest Ophthalmol Vis Sci 41: 3249-3255, 2000.

12. LI H, Xu R, Chen J and Ge F: Clinical observation of Pingmu decocion-II in treating infiltrative exophthalmos in non-active Graves' disease. Shanghai J Tradit Chin Med 42: 50-52, 2008.

13. Tomlinson JW, Durrani OM, Bujalska IJ, et al: The role of 11beta-hydroxysteroid dehydrogenase 1 in adipogenesis in thyroid-associated ophthalmopathy. J Clin Endocrinol Metab 95: 398-406, 2010.

14. Wiersinga WM and Prummel MF: Graves' ophthalmopathy: a rational approach to treatment. Trends Endocrinol Metab 13: 280-287, 2002. 